\title{
Zur Theorie der Formen vierter Ordnung.
}

\author{
Von B. Igel in Wien. ${ }^{1}$ )
}

\section{s. 1.}

In keiner Theorie in der Mathematik gibt es etwas, was sich so fertig und nach allen Richtungen hin so schön abgerundet repräsentiert, wie das Capitel "Formen vierter Ordnung." in der Theorie der binären Formen. "Und wenn ich auf dasselbe dennoch zurückkomme, so geschieht es aus folgenden drei Gründen. Es ist seit Cayley bekannt, dass die invariantentheoretische Auflösung der Gleichung vierten Grades sich auf die fundamentale Formel :

$$
\tau^{2}=-\frac{1}{2}\left\{H^{3}-\frac{i}{2} H f+\frac{j}{3} f^{3}\right\}
$$

gründet. Adjungiert man die Wurzeln der Resolvente dritten Grades, so stellt sich diese Formel in folgender Gestalt dar: .

$$
\tau^{2}=-\frac{1}{2}\left(H+e_{1} f\right)\left(H+e_{2} f\right)\left(H+e_{3} f\right)
$$

und man schließt daraus, dass die quadratischen Formen

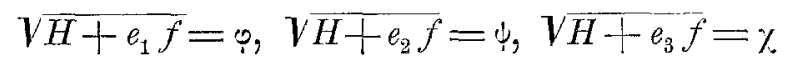

conjugierte Formen sind. Nun hat sich in neuester Zeit das Bestreben geltend gemacht, ${ }^{2}$ ) direct nachzuweisen, dass die Formen (3) conjugiert sind und daraus die Formel (2) abzuleiten. Ferner habe ich in meiner Monographie "Über die associierten Formen und deren Anwendung in der Theorie der Gleichungen" gezeigt, dass

$$
\tau(-f)=\tau . \Phi
$$

1) Herr Dr. Benzion Igel, welcher an diesen "Monatsheften" seit dem Inslebentreten derselben sich betheiligte, ist im August 1898 gestorben. Die Schriftleitung wird ihn stets in freundlicher Erinnerung behalten. Die vorliegende Arbeit, welche am 28. Mai 1893 vollendet wurde, wurde der Schriftleitung von seiner Witwe, Frau Susanne Igel, zur Veröfentlichung ïbergeben.

$\left.{ }^{2}\right)$ S. den $\$ 5$ in der Abh. des Herrn Frobenius, "Theorie der biquadr. Formen" Kronecker's Journal Bd. 106. 
wo

$$
f=\frac{\partial E}{\partial x}: \frac{\partial F}{\partial y}
$$

ist und daraus geschlossen, dass $\tau=0$ die Wurzeln hat:

$$
\left\{\begin{array}{l}
\alpha_{1}-\left[\frac{\partial F}{\partial x}: \frac{\partial F}{\partial y}\right]_{\alpha} \\
\ddot{1}_{1}-\left[\frac{\partial F}{\partial x}: \frac{\partial F}{\partial y}\right]_{\gamma} \\
\lambda_{1}-\left[\frac{\partial E}{\partial x}: \frac{\partial F}{\partial y}\right]_{\gamma}
\end{array}\right.
$$

Es entsteht nun die Frage, welche ich schon dort angedeutet habe, ob es noch Formen gibt, deren Covariante $\tau=0$ eine solche Abel'sche Gleichung sei. Endlich lehnt sich die geometrische Interpretation der Form vierter Ordnung, welche den Endpunkt des genannten Capitels bildet, gewöhnlich an die kanonische Darstellung derselben an, und es drängt sich die Erage auf, ob man nicht direct die geometrischen Sätze ableiten könnte. Ich mache im Folgenden den Endpunkt zum Anfangspunkt, d. h. ich gehe von der geometrischen Interpletation aus, beweise die geometrischen Sätze direet, beweise dann, dass die Formen $\varphi, \downarrow, \chi$ conjugiert sind and komme dann zur Relation (2).

\section{ร. 2.}

Sind $\varphi, \dot{\varphi}, \chi$ die quadratischen Factoren ron $\tau$ und sind $\alpha, \beta$; $\gamma, \delta ; u, v$ die Verschwindungselemente derselben, so bestehen, wie aus (5) folgt, die Gleichungen

$$
\left\{\begin{array}{l}
-\left[\frac{\partial F}{\partial y}: \frac{\partial F}{\partial x}\right]_{\alpha}=\beta-\left[\frac{\partial F}{\partial y}: \frac{\partial F}{\partial x}\right]_{\beta}=\alpha \\
-\left[\frac{\partial F}{\partial y}: \frac{\partial F}{\partial x}\right]_{\gamma}=\delta-\left[\frac{\partial F}{\partial y}: \frac{\partial F}{\partial x}\right]_{\delta}=\gamma \\
-\left[\frac{\partial F}{\partial y}: \frac{\partial F}{\partial x}\right]_{u}=v-\left[\frac{\partial F}{\partial y}: \frac{\partial F}{\partial x}\right]_{v}=\mu .
\end{array}\right.
$$

Aus diesen Gleichungen folgt unmittelbar, dass $F$ aus den linearen Factoren von $\tau$ auf irgend eine Weise zusammengesetzt sein müsse. Anderseits folgt aber aus dem Umstande, dass schon zwei Paare der Gleichungen (6) genügen, um $F$ zu bestimmen, dass man $F$ durch irgend zwei der quadratischen Formen darstellen kann. Wir können also auf dreierlei Weise $\not F$ darstellen:

$$
\left\{\begin{array}{l}
F=A \varphi^{2}-B \iota^{2} \\
F=A^{\prime} \varphi^{2}+B^{\prime} \chi^{2} \\
F=A^{\prime \prime} \iota^{2}+B^{\prime \prime} \chi^{2}
\end{array}\right.
$$


Aus diesen Darstellungen folgen die Gleichungen:

$$
\left\{\begin{array}{l}
\left(A-A^{\prime}\right) \varphi^{2}+b \psi^{2}-B^{\prime} \chi^{2}=0 \\
A \varphi^{2}+\left(B-A^{\prime \prime}\right) \psi^{2}-B^{\prime \prime} \chi^{2}=0 \\
A^{\prime} \varphi^{2}-A^{\prime \prime} \psi^{2}+\left(B^{\prime}-B^{\prime \prime}\right) \chi^{2}=0
\end{array}\right.
$$

und aus diesen die Gleichungen:

(9) $\left\{\begin{array}{l}\left\{B^{\prime \prime}\left(A-A^{\prime}\right)-B^{\prime} A^{\prime} \stackrel{\varphi}{ }^{2}+\left\{B B^{\prime \prime}-B^{\prime}\left(B-A^{\prime \prime}\right)\right\} \psi^{2}=0\right. \\ \left\{\left(A-A^{\prime}\right)\left(B^{\prime}-B^{\prime \prime}\right)+A^{\prime} B^{\prime \prime}\right\} \varphi^{2}+\left\{B\left(B^{\prime}-B^{\prime \prime}\right)-A^{\prime \prime} B^{\prime \prime}\right\} \psi^{2}=0 \\ \left\{A\left(B^{\prime}-B^{\prime \prime}\right)+A^{\prime} B^{\prime \prime}\right\} \varphi^{2}+\left\{\left(B-A^{\prime \prime}\right)\left(B^{\prime}-B^{\prime \prime}\right)-A^{\prime \prime} B^{\prime \prime}\right\} \psi^{2}=0 .\end{array}\right.$

Diese Coefficienten, gleich Null gesetzt, ergeben die zwei Gleichungen :

$$
\begin{aligned}
& B\left(B^{\prime}-B^{\prime \prime}\right)=A^{\prime \prime} B^{\prime} \\
& A\left(B^{\prime}-B^{\prime \prime}\right)=-A^{\prime} B^{\prime \prime}
\end{aligned}
$$

oder

$$
\frac{A}{B}=-\frac{A^{\prime}}{A^{\prime \prime} B^{\prime \prime}} .
$$

Wählt man nun $B$ so, dass es $A$ proportional ist, was immer möglich ist, so folgt, dass auch $B^{\prime}$ und $B^{\prime \prime}$ resp. $A^{\prime}$ and $A^{\prime \prime}$ proportional sind and dass man $B, B^{\prime}, B^{\prime \prime}$ mit negativen Zeichen zu nehmen hat.

Es ist daher

$$
\left\{\begin{array}{l}
F=A\left(\varphi^{2}-\psi^{2}\right) \\
F=A^{\prime}\left(\varphi^{2}-\gamma^{2}\right) \\
F=A^{\prime \prime}\left(\psi^{2}-\chi^{2}\right)
\end{array}\right.
$$

Von den Größen $A^{\prime}$, welche für diesen §. irrelevant sind und im $\$ .3$ bestimmt werden, wollen wir jetzt absehen. Auch wollen wir nur e in e dieser Darstellungen von $F$ zu Grunde legen und zwar möge es die erste sein. Aus derselben folgt:

$$
\begin{gathered}
\frac{\partial F}{\partial y}: \frac{\partial F}{\partial x}= \\
=\frac{-\alpha(x-\alpha y)(x-\beta y)^{2}-\beta(x-\alpha y)^{2}(x-\beta y)+\gamma(x-\gamma y)(x-\delta y)^{2}+\delta(x-\gamma y)^{2}(x-\delta y)}{(x-\alpha y)(x-\beta y)^{2}+(x-\alpha y)^{2}(x-\beta y)-(x-\gamma y)(x-\delta y)^{2}+(x-\gamma y)^{2}(x-\delta y)}
\end{gathered}
$$

folglich ist

$$
\left\{\begin{array}{l}
{\left[\frac{\partial F}{\partial y}: \frac{\partial F}{\partial x}\right]_{\alpha}=\frac{\gamma(\alpha-\gamma)(\alpha-\delta)^{2}+\delta(\alpha-\gamma)^{2}(\alpha-\delta)}{-(\alpha-\gamma)(\alpha-\delta)^{2}-(\alpha-\gamma)^{2}(\alpha-\delta)}=-\beta} \\
{\left[\frac{\partial F}{\partial y}: \frac{\partial F}{\partial x}\right]_{\gamma}=\frac{-\alpha(\gamma-\alpha)(\gamma-\beta)^{2}-\beta(\gamma-\alpha)^{2}(\gamma-\beta)}{(\gamma-\alpha)(\gamma-\beta)^{2}+(\gamma-\alpha)^{2}(\gamma-\beta)}=-\delta .}
\end{array}\right.
$$


Entwickelt geben beide dieselbe Gleichung:

$$
(\gamma-\alpha)(\gamma-\beta)\{(\delta-\alpha)(\gamma-\beta)+(\delta-\beta)(\gamma-\alpha)\}=0,
$$

d. h. die Elementenpaare $\alpha, 3 ; \gamma, \delta$ sind $z u$ einander harmonisch. Es ist ferner

$$
\begin{aligned}
& \qquad\left[\frac{\partial F}{\partial y}: \frac{\partial F}{\partial x}\right]_{\mu}= \\
& =\frac{-\alpha(\mu-\alpha)(\mu-\beta)^{2}-\beta(\mu-\alpha)^{2}(\mu-\beta)+\gamma(\mu-\gamma)(\mu-\delta)^{2}+\delta(\mu-\gamma)^{2}(\mu-\delta)}{(\mu-\alpha)(\mu-\beta)^{2}+(\mu-\alpha)^{2}(\mu-\beta)-(\mu-\gamma)(\mu-\delta)^{2}-(\mu-\gamma)^{2}(\mu-\delta)}=-\nu .
\end{aligned}
$$

Entwickelt gibt dies die Gleichung

$$
\begin{aligned}
& (\nu-\alpha)(\mu-\alpha)(\mu-\beta)^{2}+(\nu-\beta)(\mu-\alpha)^{2}(\mu-\beta)+ \\
& +(\nu-\gamma)(\mu-\gamma)(\mu-\delta)^{2}+(\nu-\delta)(\mu-\gamma)^{2}(\mu-\delta)== \\
& =(\mu-\alpha)(\mu-\beta)\{\nu-\alpha)(\mu-\beta)+(\nu-\beta)(\mu-\alpha)\}+ \\
& +(\mu-\gamma)(\mu-\delta)\{(\nu-\gamma)(\mu-\delta)+(\nu-\delta)(\mu-\gamma)\}=0 .
\end{aligned}
$$

Vertauscht man $\mu$ mit $\nu$, so ändern sich die Ausdrücke innerhalb der Klammern nicht, während die außerhalb derselben sich ändern. Wir schließen daher, dass (14) in folgende zwei Relationen zerfällt :

$$
\left\{\begin{array}{l}
(\nu-\alpha)(\mu-\beta)+(\nu-\beta)(\mu-\alpha)=0 \\
(\nu-\gamma)(\mu-\delta)+(\nu-\delta)(\mu-\gamma)=0 .
\end{array}\right.
$$

Die Gleichungen (13) und (15) liefern den bekannten Satz: "Die drei quadratischen Factoren von $\tau$ stellen drei Elementenpaare dar, von denen je zwei zu einander harmonisch sind."

Zerlegen wir $F$ in seine linearen Factoren, setzen also

$$
F=(x-A y)(x-B y)(x-\Gamma y)(x-\Delta y)
$$

so folgen aus (6) die drei Gleichungen

$$
\left\{\begin{array}{c}
(\beta-A)(\alpha-B)(\alpha-\Gamma)(\alpha-\Delta)+ \\
+(\beta-B)(\alpha-A)(\alpha-\Gamma)(\alpha-\Delta)+ \\
+(\beta-\Gamma)(\alpha-A)(\alpha-B)(\alpha-\Delta)+ \\
+(\beta-\Delta)(\alpha-A)(\alpha-B)(\alpha-\Gamma)=0 \\
(\delta-A)(\gamma-B)(\gamma-\Gamma)(\gamma-\Delta)+ \\
+(\delta-B)(\gamma-A)(\gamma-\Gamma)(\gamma-\Delta)+ \\
+(\delta-\Gamma)(\gamma-A)(\gamma-B)(\gamma-\Delta)+ \\
+(\delta-\Delta)(\gamma-A)(\gamma-B)(\gamma-\Gamma)=0 \\
(\nu-A)(\mu-B)(\mu-\Gamma)(\mu-\Delta)+ \\
+(\nu-B)(\mu-A)(\mu-\Gamma)(\mu-\Delta)+ \\
+(\nu-\Gamma)(\mu-A)(\mu-B)(\mu-\Delta)+ \\
+(\nu-\Delta)(\mu-A)(\mu-B)(\mu-\Gamma)=0 .
\end{array}\right.
$$


Dieselben lassen sich folgendermaßen schreiben:

$$
\left\{\begin{array}{c}
(\alpha-\Gamma)(\alpha-\Delta)\{(\beta-A)(\alpha-B)+(\beta-B)(\alpha-A)\} \\
+(\alpha-A)(\alpha-B)\{(\beta-\Gamma)(\alpha-\Delta)+(\beta-\Delta)(\alpha-\Gamma)\}=0 \\
(\gamma-B)(\gamma-\Delta)\{(\gamma-A)(\gamma-\Gamma)+(\gamma-\Gamma)(\gamma-\Delta)\} \\
+(\gamma-A)(\gamma-1)\{(\gamma-B)(\gamma-\Delta)+(\gamma-\Delta)(\gamma-B)\}=0 \\
\quad(\mu-B)(\mu-\Gamma)\{(\nu-A)(\mu-\Delta)+(\nu-\Delta)(\mu-A)\} \\
+(\mu-A)(\mu-A)\{(\nu-B)(\mu-\Gamma)+(\nu-\Gamma)(\mu-B)\}=0 .
\end{array}\right.
$$

Aus dem Umstande, dass bei einer Vertauschung von $A$ mit $B$ und $\Gamma$ mit $\Delta$ die Ausdrücke innerhalb der Klammern sich nicht ändern, während diejenigen außerhalb der Klammern sich ändern. schließt man, wie oben, dass jede der Gleichungen in zwei zerfällt Die aus diesen folgenden Gleichungen

$$
\begin{array}{ll}
\frac{(\beta-A)(\alpha-B)}{(\beta-B)(\alpha-A)}=-1, & \frac{(\beta-\Gamma)(\alpha-\Delta)}{(\beta-\Delta)(\alpha-\Gamma)}=-1, \\
\frac{(\delta-A)(\gamma-\Gamma)}{(\delta-\Gamma)(\gamma-A)}=-1, & \frac{(\gamma-B)(\gamma-\Delta)}{(\gamma-\Delta)(\gamma-B)}=-1, \\
\frac{(\nu-A)(\mu-\Delta)}{(\nu-\Delta)(\mu-A)}=-1, & \frac{(\nu-B)(\mu-\Gamma)}{(\nu-\Gamma)(\mu-B)}=-1
\end{array}
$$

liefern den bekannten Satz:

"In Bezug auf die Elemente eines jeden der Factorer von $\tau$ zerlegt sich die Form $F$ in zwei Elementenpaare welche zu jenen beiden harmonisch sind, so dass den dre quadratischen Factoren von $\tau$ die drei Zerlegungen der vies Elemente von $F=0$ entsprechen."

Bildet man die Form

$$
\Phi=\varphi^{2}-k \iota^{2}
$$

so stellt dieselbe eine einfach unendliche Schaar von Quadrupelı dar, wenn man $k$ alle möglichen Werte beilegt. Für alle in d enthaltenen Formen gelten offenbar die Gleichungen

$$
\begin{cases}-\left[\frac{\partial \Phi}{\partial y}: \frac{\partial \Phi}{\partial x}\right]_{\alpha}=\beta & -\left[\frac{\partial \Phi}{\partial y}: \frac{\partial \Phi}{\partial x}\right]_{\beta}=x \\ -\left[\frac{\partial \Phi}{\partial y}: \frac{\partial \Phi}{\partial x}\right]_{\gamma}=\delta & -\left[\frac{\partial \Phi}{\partial y}: \frac{\partial \Phi}{\partial x}\right]_{\delta}=\gamma \\ -\left[\frac{\partial \Phi}{\partial y}: \frac{\partial \Phi}{\partial x}\right]_{\mu}=\nu & -\left[\frac{\partial \Phi}{\partial y}: \frac{\partial \Phi}{\partial x}\right]_{\nu}=\gamma .\end{cases}
$$

Es folgt daher der bekannte Satz: "Jedes Quadrupel der Schaar

$$
\varphi^{2}-k \psi^{2}=0
$$


zerlegt sich in Bezug auf die Elemente eines jeden der Factoren von $\tau$ in zwei Elementenpare, welche zu jenen beiden harmonisch sind, so dass den drei quadratischen Factoren von $\tau$ die drei Zerlegungen der vier Elemente von $\varphi^{2}-k \psi^{2}$ entsprechen." beweisen:

Es erübrigt nun noch, den folgenden bekannten Satz zu "Ist ein System von drei zu einander harmonischen Elementenpaaren gegeben, so bilden alle Quadrupel, deren verschiedene Zerlegungen jedesmal zwei zu einem der gegebenen harmonische Paare liefern, eine Schaar $\varphi^{2}-k^{2} \psi^{2}=0$, für welche die zugehörige Gleichung $\tau=0$ die gegebenen drei Paare sind."

Dieser Satz lässt sich in folgenden umsetzen:

Wenn eine Form sechster Ordnung $F(x, y)$ sich auf folgende Weise zerlegen lässt

$$
F=\left(x-\alpha^{\prime} y\right)\left(x-\Theta\left(\alpha^{\prime}\right) y\right)\left(x-\gamma^{\prime} y\right)\left(x-\Theta\left(\gamma^{\prime}\right) y\right)\left(x-\mu^{\prime} y\right)\left(x-\Theta\left(u^{\prime}\right) y\right),
$$

wo

$$
\Theta=-\frac{\partial \Phi}{\partial y}: \frac{\partial \Phi}{\partial x}
$$

und $\Phi$ eine Form vierter Ordnung ist, so ist $F$ die Covariante $\tau$ der Form $\Phi$.

Beweis.

Für die Form $\tau$ von $\Phi$ bestehen die Gleichungen (13), (15) und (16). Für $F$ bestehen der Annahme nach ebenfalls solche Gleichungen, welche aus jenen hervorgehen, wenn man an Stelle von $\alpha, \gamma, \mu, x^{\prime}, \gamma^{\prime}, \mu^{\prime}$ setzt. Wäre nun $F$ nicht identisch mit $\tau$, d. b. fielen die genannten Größen nicht zusammen, so wïrde folgen, dass es zu zwei Punktepaaren von $\Phi=0$ zwei Punktepaare gibt, welche zu jenen harmonisch sind, was bekanntiich nicht der Fall sein kann; es folgt daher, dass $F$ mit $\tau$ identisch ist.

\section{\$. 3.}

Wir gehen nun dazu über, zu zeigen, wie aus den Gleichungen (6) in $\$$. 2 die übrigen Eigenschaften von $\tau$, von denen jede, wie (l. c.) nachgewiesen wurde, characteristisch ist, sich ergeben. Zunächst wollen wir zeigen, dass $\vartheta, \downarrow, \%$ conjugierte Formen sind, das heißt, dass die simultanen Invarianten, von je zwei gebildet, verschwinden. Zu diesem Zwecke bemerken wir, dass $H$ in der Schaar

$$
\varphi^{2}-k \dot{\varphi}^{2}
$$

enthalten ist. Es lrommt also darauf an, den Parameter $k$ zu bestimmen, für welchen die Form $\varphi^{2}-k \psi^{2}$ das $H$ vorstellt. Bilden 
wir die Hesse'sche Determinante der Form $\varphi^{2}-\mathrm{w}^{2}$, so ist dieselbe

$$
\begin{aligned}
& H=\left|\begin{array}{ll}
\frac{\partial^{2}\left(\varphi^{2}-\psi^{2}\right)}{\partial x^{2}} & \frac{\partial^{2}\left(\varphi^{2}-\psi^{2}\right)}{\partial x \partial y} \\
\frac{\partial^{2}\left(\varphi^{2}-\psi^{2}\right)}{\partial x \partial y} & \frac{\partial^{2}\left(\varphi^{2}-\varphi^{2}\right)}{\partial y^{2}}
\end{array}\right| \frac{1}{3^{2} \cdot 4^{2}}
\end{aligned}
$$

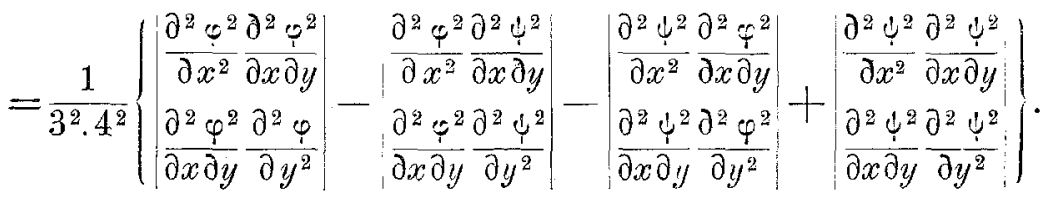

Wertet man die Determinanten innerhalb der Klammer aus, so erbält man die Formel:

$$
\begin{gathered}
\frac{1}{3^{2}}\left\{3 H(\varphi) \varphi^{2}-+3 H(\psi) \psi^{2}-5(\varphi \psi)_{2} \varphi \varphi+2 H(\psi) \varphi^{2}+2 H(\varphi) \psi^{2}-\right. \\
\left.-4 J(\varphi \psi)^{2}\right\}=H(F) .
\end{gathered}
$$

Diese Formel führt nun auf folgende Sätze:

I. Die Formen $\varphi, \downarrow$ sind conjugiert, d. h. ihre zweite Überschiebung über einander verschwindet.

II. Das Quadrat der Jacobischen Determinante von $\varphi$ und 4 lässt sich durch die Summe der Quadrate dieser Formen darstellen.

Da bekanntlich die Identität besteht:

$$
J(\varphi \psi)^{2}=-\frac{1}{2}\left\{H(\varphi) \psi^{2}-2(\varphi \psi)_{2} \varphi \psi+H(\psi) \varphi^{2}\right\}
$$

so können sich in (20) die Producte $\varphi \cdot 4$ nicht wegheben; da aber infolge der obigen Bemerkung $H\left(F^{\dagger}\right)$ sich durch die Quadrate dieser darstellen lassen muss, so müssen die Identitäten bestehen:

$$
\left\{\begin{array}{l}
(\varphi \circlearrowleft)_{2}=0 \\
J(\varphi \downarrow)^{2}=a \varphi^{2}+b \psi^{2} .
\end{array}\right.
$$

Um die endgiltige Form $H(F)$ zu erhalten, erinnere man sich an folgende Sätze: Das Verschwinden der drei simultanen Invarianten

$$
(\div)_{2},(c \%),(\omega, \gamma)
$$

bat zur Folge, dass die Functionaldeterminanten

$$
J(\rho)=(\varphi \varphi)_{1}, \quad J(\% \varphi)=(\chi \varphi)_{1}, \quad J(\psi \gamma)=(\psi \%)_{1}
$$

den Originalformen proportional werden, dass also die Identitäten bestehen :

$$
(\psi \%)_{1}=\lambda \varphi, \quad(\% \varphi)_{1}=\mu \psi, \quad(\varphi \psi)_{1}=\nu \% .
$$


Aus diesen Identitäten folgt ferner mit Berücksichtigung einer bekannten Identität die Identität

$$
\lambda \varphi^{2}+\mu \iota^{2} \frac{1}{1} v \chi^{2}=0
$$

Endlich folgt aus der bekannten Identität

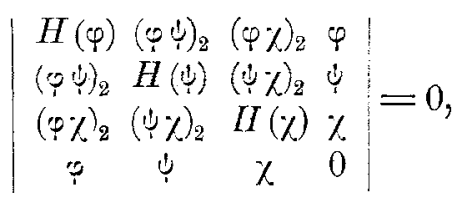

welche in unserem Falle in folgende übergeht:

die Gleichung

$$
H(\downarrow) H(\chi) \varphi^{2}+H(\varphi) H(\chi) \psi^{2}+H(\varphi) H(\varphi) \chi^{2}=0
$$

$$
H(\biguplus) H(\gamma)=\rho \lambda, \quad H(\varphi) H(\gamma)=\rho \mu, \quad H(\varphi) H(\psi)=\rho v .
$$

Die Gleichung (20) lässt sich jetzt folgendermaßen schreiben:

$$
\begin{aligned}
& H(F)=\frac{1}{3^{2}}\left\{3 H(\vartheta) \varphi^{2}+2 H(\psi) \varphi^{2}+3 H(\psi) \varphi^{2}+2 H(p) \varphi^{2}+\right. \\
& \left.+{ }_{\rho^{2}}^{4} H(\varphi) H(\psi)^{2} H(\chi) \varphi^{2}+\frac{4}{\rho^{2}} H(\varphi)^{2} H(\psi) H(\chi) \psi^{2}\right\} \\
& =\frac{1}{3^{2}}\left\{3 H(\varphi)+2 H(\downarrow)+\frac{4}{\rho^{2}} H(\varphi) H(\downarrow)^{2} H(\alpha)\right\} \varphi^{2}+ \\
& +\frac{1}{3^{2}}\left\{3 H(\varphi)+2 H(\varphi)+\frac{4}{p^{2}} H(\varphi)^{2} H(\varphi) H(\chi)\right\} \iota^{2} .
\end{aligned}
$$

Diese Formel gibt uns nun Aufschluss über die Formen $H(\varphi)$, $H(\psi), H(\chi)$ und über die Größe $p$.

Der Character von $H(F)$ als Covariante verträgt es offenbar nicht, dass in ihr Glieder von verschiedenem Grade in den Coefficienten vorkommen, es muss daher $\rho$ ebenfalls von den Coefficienten der drei Formen abhängen und zwar so, dass die Ausdrücke

$$
\begin{aligned}
& \frac{1}{p^{2}} H(\varphi) H(\downarrow)^{2} H(\chi) \\
& \frac{1}{p^{2}} H(\varphi)^{2} H(\psi) H(\chi)
\end{aligned}
$$

nur Glieder rom zweiten Grade geben. In der That ergibt die Formel

den Wert von $p$

$$
J\left(\varphi(\psi)^{2}=\nu^{2} \chi^{2}=-\left\{H(\varphi) \psi^{2}+H(\varphi) \varphi^{2}\right\}\right.
$$

$$
\rho=\sqrt{H(\varphi) H(\psi) H(\chi)}
$$

Überlegt man, dass $i, \mu, v$ offenbar nicht irrational sein können und dass

$$
H(\varphi), \quad H(\psi), \quad H(\gamma)
$$


nicht vollständige Quadrate sind, so schließt man, dass diese Invarianten sich aus denselben drei Größen in Gestalt von Producten aus je zwei Summen oder je zwei Differenzen ausdrücken lassen, so dass

ist und

$$
\begin{aligned}
& H(\varphi)=A\left(k \pm k_{1}\right)\left(k \pm k_{2}\right) \\
& H(\psi)=A\left(k_{1} \pm k_{2}\right)\left(k_{1} \pm k\right) \\
& H(\chi)=A\left(k_{2} \pm k_{1}\right)\left(k_{2} \pm k\right)
\end{aligned}
$$

$$
\rho=D \cdot\left(k_{ \pm} \pm k_{1}\right)\left(k_{1} \pm k_{2}\right)\left(k_{2} \pm k\right) \text {. }
$$

Es ist alsdann

$$
\begin{aligned}
& \frac{1}{\rho^{2}} H(\varphi) H(\biguplus)^{2} H(\chi)=-\frac{A^{4}}{D^{2}}\left(k \pm k_{1}\right)\left(k_{2} \pm k_{1}\right) \\
& \frac{1}{\rho^{2}} H(\varphi)^{2} H(\biguplus) H(\chi)=-\frac{A^{4}}{D^{2}}\left(k_{1} \pm k\right)\left(k_{2} \pm k\right) .
\end{aligned}
$$

Wenn nun zur Abkürzung

$$
A^{4}: D^{2}=C
$$

gesetzt wird, so geht die Formel (22) in folgende über:

$$
\begin{aligned}
& H(F)=\frac{\vartheta^{2}}{3^{2}}\left\{3 A\left(k \pm k_{1}\right)\left(k \pm k_{2}\right)+2 A\left(k_{1} \pm k_{2}\right)\left(k_{1} \pm k\right)-\right. \\
& \left.-4 C\left(k_{i} \pm k_{1}\right)\left(k_{2} \pm k_{1}\right)\right\}+ \\
& +\frac{y^{2}}{3^{2}}\left\{3 A\left(k_{1} \pm k_{2}\right)\left(k_{1} \pm k\right)+2 A\left(k \pm k_{1}\right)\left(k \pm k_{2}\right)-\right. \\
& \left.-4 C\left(k_{1} \pm k\right)\left(k_{2} \pm k\right)\right\} \\
& =\frac{\left(k_{1} \pm k\right)}{3^{2}}\left\{ \pm 3 A\left(k \pm k_{2}\right)+2 A\left(k_{1} \pm k_{2}\right) \mp 4 C\left(k_{2} \pm k_{1}\right)\right)_{\rho} \rho^{2}+ \\
& \left.+\frac{\left(k_{1} \pm k\right)}{3^{2}}\left\{3 A\left(k_{1} \pm k_{2}\right) \pm 2 A\left(k \pm k_{2}\right)-4 C\left(k_{2} \pm k\right)\right\}\right\}^{2} .
\end{aligned}
$$

Um nun zu entscheiden, welches von den Doppelzeichen zu nehmen ist, muss man die Bedeutung der Größon $k_{i}$ aufsuchen. Dieselbe findet man durch folgende Überlegung. Durch Adjungierung der Wurzeln der Gleichung

$$
\left|\begin{array}{ccc}
a_{0} & a_{1} & a_{2}-k \\
a_{1} & a_{2}+\frac{k}{2} & a_{3} \\
a_{2}-k & a_{3} & a_{4}
\end{array}\right|=-\frac{1}{2}\left(k^{3}-\frac{i}{2} k-\frac{j}{3}\right)=0
$$

lässt sich die Form $F$ auf dreierlei Weise in zwei quadratische Factoren zerlegen. Es müssen daher, da

$$
\begin{aligned}
F & =A(\varphi-\psi)(\varphi+\psi) \\
& =A^{\prime}(\chi-\varphi)(\chi+\varphi) \\
& =A^{\prime \prime}(\psi-\psi)(\psi+\gamma)
\end{aligned}
$$


offenbar diese drei Zerlegungen sind, die $h$ die Wurzeln der Gleichung (24) sein. Die Aggregate in den Klammern von (23) müssen einzeln durch $3^{2}$ theilbar sein, und zwar muss sich jeder Factor, der mit irgend einem $k$ multipliciert ist, durch $3^{2}$ theilen lassen. Das Pluszeichen ist demnach zu verwerfen, da man unter dessen Annahme unmöglich die Größen $A$ und $C$ so bestimmen kann, dass die Klammerfactoren durch $3^{2}$ theilbar seien. Hingegen geht die Formel (23), wenn man das Zeichen - nimmt und die Relation

$$
k+k_{1}+k_{2}=0
$$

beriucksichtigt, in folgende über:

$$
\begin{aligned}
H(F) & =\frac{\left(k_{1}-k\right)}{3^{2}}\left\{(5 A-4 C) k_{1}+(4 A+4 C) k_{2}\right\} \varphi^{2}+ \\
& +\frac{\left(k_{1}-k\right)}{3^{2}}\left\{(4 A+4 C) k_{1}+(-A+8 C) k_{1} \iota^{2} .\right.
\end{aligned}
$$

so muss

Sollen nun diese Klammerfactoren durch $3^{2}$ theilbar sein

$$
A=-\frac{1}{2}, \quad C=\frac{1}{2}
$$

sein, so dass wir die Formel erhalten

$$
H(F)=-\frac{\left(k_{1}-k\right)}{2}\left(k_{1} \oplus^{2}-k \iota^{2}\right) \text {. }
$$

Bei der Ableitung dieser Formel sind die Constanten $A^{i}$ vernachlässigt worden; wie man diese bestimmt und wie sich alsdann die Formel modificiert, wird im nächsten §. gezeigt werden.

\section{\$. 4.}

In der Schaar

$$
\varphi^{2}-k \varphi^{2}
$$

gibt es so viele Formen, welche Doppelfactoren besitzen, als die Anzahl der linearen Factoren der Jacobi'schen Determinante beträgt. Die entsprechenden $k$ bestimmen sich bekanntlich durch die Gleichung, welche entsteht, wenn man die Discriminante der Schaar gleich Null setzt. Nun ist

$$
J\left(\psi^{2} \cdot \varphi^{2}\right)=2 \varphi \cdot \psi(\varphi, \varphi)_{1}=2 \varphi \cdot \varphi \cdot \psi
$$

es sind daher die Doppelfactoren Factoren resp. von

$$
\varphi, \biguplus, \chi \text {. }
$$

Dem Character unserer Schaar gemäß muss diejenige Form, die einen der Factoren einer der conjugierten Formen doppelt 
enthält, auch den anderen Factor derselben doppelt enthalten. Es folgt daher, dass die Discriminante der Schaar ein vollständiges Quadrat ist. Um die Gestalt derselben zu ermitteln, bemerken wir, dass den Formen $\varphi^{2}, \bigcup^{2}$ die Werte Null und Unendlich von $k$ entsprechen. Daraus und mit Berüeksichtigung einer schon oft erwähnten Formel folgt unmittelbar, dass die Gestalt der Discriminante folgende ist:

(27) Diserim. $\left(\varphi^{2}-k \psi^{2}\right)=C \cdot \lambda^{2}\{H(\psi) H(\chi) \lambda+H(\varphi) H(\chi)\}^{2}$.

Aus dieser Formel ergibt sich nun die Discriminante der Form

$$
F=A\left(\varphi^{2}-\psi^{2}\right)
$$

$$
\text { Discrim. }(F)=c A^{2}\{H(\psi) H(\chi)+H(\varphi) H(\chi)\}^{2} \text {. }
$$

Setzen wir die Schaar aus $F$ und $H$ zusammen, bilden also die Form

$$
H+\lambda F
$$

und fragen nach denjenigen Formen, welche lineare Factoren der Functionaldeterminante von $F$ und $H$ als Doppelfactoren besitzen, so wissen wir, dass dieselben $\varphi^{2}, \cup^{2}, \chi^{2}$ sind.

Daraus resultiert zunächst, dass die Diseriminante von $H+\lambda . F^{\text {, }}$ von einem von $\lambda$ unabhängigem Factor abgesehen, das Quadrat einer cubischen Form in $\lambda$ darstellt. Bezeichnen wir dieselbe mit $\Omega(\lambda)$, so ist also

Discrim. $(H+\lambda . F)=M . \Omega(\lambda)^{2}$.

Es handelt sich jetzt darum, diese cubische Form herzustellen und dazu führt folgende Überlegung. Sind die Verschwindungselemente derselben $\lambda_{1}, \lambda_{2}, \lambda_{3}$ und ist etwa

$$
\left(k_{1} \varphi^{2}+k \psi^{2}\right)+\lambda\left(\varphi^{2}-\psi^{2}\right)=C \varphi^{2}
$$

so muss offenbar $k=\lambda$, sein. Da nun $k$ die Wurzeln der Lagrange'schen Resolvente sind, so folgt

$$
\Omega(\lambda)=B \cdot\left(\lambda^{3}-\frac{i}{2} \lambda-\frac{j}{3}\right) .
$$

Jetzt sind wir nun in der Lage, mit Hilfe der Formel (26) die Constante $A$ in

$$
F=A\left(\varphi^{2}-\psi^{2}\right)
$$

zu bestimmen. Da nämlich $A$ in $H(F)$ im Quadrat erscheint, so ist es nur dann möglich, dass

ist, wenn

$$
H(F)+k F=C \varphi^{2}
$$

$$
A=-\frac{2}{k-k_{1}} \text {. }
$$


Wir erhalten daher die Formeln

$$
\left\{\begin{array}{l}
F=-\frac{2}{k-k_{1}}\left(\varphi^{2}-\psi^{2}\right) \\
H(F)=-\frac{2}{k_{1}-k}\left(k_{1} \varphi^{2}-k \psi^{2}\right)
\end{array}\right.
$$

(32) $\left\{\begin{array}{l}H+k F=-\frac{2}{k_{1}-k}\left(k_{1} \varphi^{2}-k \psi^{2}\right)-\frac{2 k}{k-k_{1}}\left(\varphi^{2}-\omega^{2}\right)=-2 \varphi^{2} \\ H+k_{1} F=-\frac{2}{k_{1}-k}\left(k_{1} \varphi^{2}-k \psi^{2}\right)-\frac{2 k_{1}}{k-k_{1}}\left(\varphi^{2}-\psi^{2}\right)=-2 \psi^{2} \\ H+k_{2} F=-\frac{2}{k_{1}-k}\left(k_{1} \varphi^{2}-k \psi^{2}\right)-\frac{2 k_{2}}{k-k_{1}}\left(\varphi^{2}-\psi^{2}\right)=-2 \chi^{2}\end{array}\right.$ Identität

Die letzte dieser Identitäten folgt aus der schon oben citierten

$$
H(\psi) H(\chi) \varphi^{2}+H(\varphi) H(\chi) \varphi^{2}+H(\varphi) H(\psi) \chi^{2}=0
$$

Da nämlich, von einem Zahlenfactor abgesehen,

$$
\begin{aligned}
& H(\zeta) H(\chi)=\left(k_{2}-k_{1}\right)\left\{\left(k-k_{1}\right)\left(k_{1}-k_{2}\right)\left(k_{2}-k\right)\right\} \\
& H(\varphi) H(\chi)=\left(k_{1}-k_{2}\right)\left\{\left(k-k_{1}\right)\left(k_{1}-k_{2}\right)\left(k_{2}-k\right)\right\} \\
& H(\wp) H(\zeta)=\left(k_{1}-k\right)\left\{\left(k-k_{1}\right)\left(k_{1}-k_{2}\right)\left(k_{2}-k\right)\right\}
\end{aligned}
$$

ist, so folgt

$$
\begin{aligned}
& -\frac{2}{k_{1}-k}\left(k_{1} \varphi^{2}-k \psi^{2}\right)-\frac{2 k_{2}}{k-k_{1}}\left(\varphi^{2}-\psi^{2}\right)=\frac{2}{k_{1}-k}\left\{\left(k_{2}-k_{1}\right) \varphi^{2}+\left(k-k_{2}\right) \biguplus^{2}\right\} \\
& \quad=-2 \chi^{2} \\
& \text { Da ferner }
\end{aligned}
$$

$$
H(\chi)^{2}\{H(\omega)+H(\varphi)\}^{2}=C \cdot\left(k-k_{1}\right)^{4}\left(k_{1}-k_{2}\right)^{2}\left(k_{2}-k\right)^{2},
$$

so folgt aus der Formel (28) die belzannte Formel

$$
\text { Discrim. }(F)=C^{\prime}\left(k-k_{1}\right)^{2}\left(k_{1}-k_{2}\right)^{2}\left(k_{2}-k\right)^{2} \text {. }
$$

Die Multiplication der drei Formeln in (32) liefert die fundamentale Formel:

$$
\tau^{2}=-\frac{1}{2}(H+k F)\left(H+k_{1} F\right)\left(H+k_{2} F\right) .
$$

\section{ร. 5.}

Es erübrigt nur noch zu zeigen, wie aus den Gleichungen in $\$$. 2 die fundamentale Eigenschaft von $\tau$, dass ibre vierte Überschiebung über sich selbst verschwindet, sich ergibt. Diese Covariante muss sich durch $F$ und $H$ und infolge dessen durch die 
conjugierten quadratischen Formen darstellen lassen. Bezeichnet man die Wurzeln von $\tau=0$ mit $\alpha_{1}, \alpha_{2}, \alpha_{3}, \alpha_{4}, a_{5}, \alpha_{6}$, drückt die Covariante $\left(\tau \tau^{\prime}\right)_{4}$ durch diese Wurzeln aus und berücksichtigt die bekannte Darstellung der Covarianten gerader Ordnung durch die Wurzeln der Grundform, nämlich

$$
\left(\tau \tau^{\prime}\right)_{4}=\tau_{0}^{e} \Sigma\left(\alpha_{1}-\alpha_{2}\right)^{\nu_{1}}\left(\alpha_{2}-\alpha_{3}\right)^{\nu_{2}} \ldots\left(x-\alpha_{1} y\right)^{\hat{\alpha}_{1}}\left(x-\alpha_{2} y\right)^{\hat{\alpha}_{2}} \ldots
$$

so siebt man sofort, dass in der Darstellung von $\left(\tau \tau^{\prime}\right)_{4}$ durch die conjugierten Formen die Quadrate derselben nicht vorkommen können. Es muss daher

$$
\left(\tau \tau^{\prime}\right)_{4}=a \varphi \psi+b \varphi \chi+c \psi \psi
$$

sein. Dann ist

$$
\begin{aligned}
& a=\left(\alpha_{5}-\alpha_{6}\right)\left(\alpha_{6}-\alpha_{3}\right)\left(\alpha_{5}-\alpha_{4}\right)\left(\alpha_{1}-\alpha_{2}\right)+ \\
& \quad+\left(\alpha_{5}-\alpha_{6}\right)\left(\alpha_{6}-\alpha_{4}\right)\left(\alpha_{5}-\alpha_{3}\right)\left(\alpha_{1}-\alpha_{2}\right)+ \\
& \quad+\left(\alpha_{5}-\alpha_{6}\right)\left(\alpha_{6}-\alpha_{1}\right)\left(\alpha_{5}-\alpha_{2}\right)\left(\alpha_{3}-\alpha_{4}\right)+ \\
& \quad+\left(\alpha_{5}-\alpha_{6}\right)\left(\alpha_{6}-\alpha_{2}\right)\left(\alpha_{5}-\alpha_{1}\right)\left(\alpha_{3}-\alpha_{4}\right) \\
& b=\left(\alpha_{3}-\alpha_{4}\right)\left(\alpha_{4}-\alpha_{5}\right)\left(\alpha_{3}-\alpha_{6}\right)\left(\alpha_{1}-\alpha_{2}\right)+ \\
& \quad+\left(\alpha_{3}-\alpha_{4}\right)\left(\alpha_{4}-\alpha_{6}\right)\left(\alpha_{3}-\alpha_{5}\right)\left(\alpha_{1}-\alpha_{2}\right)+ \\
& +\left(\alpha_{3}-\alpha_{4}\right)\left(\alpha_{4}-\alpha_{2}\right)\left(\alpha_{3}-\alpha_{1}\right)\left(\alpha_{5}-\alpha_{6}\right)+ \\
& \quad+\left(\alpha_{3}-\alpha_{4}\right)\left(\alpha_{4}-\alpha_{1}\right)\left(\alpha_{3}-\alpha_{2}\right)\left(\alpha_{5}-\alpha_{6}\right) \\
& c=\left(\alpha_{1}-\alpha_{2}\right)\left(\alpha_{2}-\alpha_{5}\right)\left(\alpha_{1}-\alpha_{6}\right)\left(\alpha_{3}-\alpha_{4}\right)+ \\
& \quad+\left(\alpha_{1}-\alpha_{2}\right)\left(\alpha_{2}-\alpha_{6}\right)\left(\alpha_{1}-\alpha_{5}\right)\left(\alpha_{3}-\alpha_{4}\right)+ \\
& \quad+\left(\alpha_{1}-\alpha_{2}\right)\left(\alpha_{2}-\alpha_{3}\right)\left(\alpha_{1}-\alpha_{4}\right)\left(\alpha_{5}-\alpha_{6}\right)+ \\
& \quad+\left(\alpha_{1}-\alpha_{2}\right)\left(\alpha_{2}-\alpha_{4}\right)\left(\alpha_{1}-\alpha_{3}\right)\left(\alpha_{5}-\alpha_{6}\right)
\end{aligned}
$$

und man sieht sofort, dass diese Coefficienten infolge der Glei chungen (6) in $\$ .2$ verschwinden. Daraus folgt

$$
\left(\tau \tau^{\prime}\right)_{t}=0 \text {. }
$$

\$. 6.

Und nun gehen wir an die Beantwortung der von uns in de citierten Monographie aufgeworfenen Frage: Wie beweist mar dass die Gleichungen höheren Grades in der Weise wie die Gle: chung vierten Grades invariantentheoretisch nicht zu lösen sinc $\mathrm{Zu}$ diesem Zwecke recapituliere ich in Kürze die Methode, welch ich dort für die Lösung der Gleichung vierten Grades gegebe habe. Aus der Formel

$$
\tau(-f)=\tau\left\{2 F^{3} j+H F i-H^{3}\right\}
$$

habe ich geschlossen, dass die Nullwerte von $~$ auch Nullwerte vc $\tau(-f)$ sein müssen, d. h. dass $\tau=0$ eine Abel'sche Gleichur 
(im engeren Sinne) sein müsse. Und dass die Periode derselben gleich 2 sei, habe ich aus der Formel:

$$
f(-f)=\frac{F \frac{\partial \tau}{\partial y}-(\tau F)_{1} \cdot x}{F \frac{\partial \tau}{\partial x}+(\tau F)_{1} \cdot y}
$$

gefolgert. Wird nämlich berücksichtigt, dass

$$
\left[\frac{\partial \tau}{\partial y}\right]_{\alpha}=-\rho \alpha,\left[\frac{\partial \tau}{\partial x}\right]_{\alpha}=p \alpha,
$$

ist, so geht diese Formel, wenn man für $x$ und $y$ resp. $\alpha_{1}$ und $\alpha_{2}$ setzt, über in

$$
[f(-f)]_{\alpha_{1}: \alpha_{2}}=\frac{\alpha_{1}}{\alpha_{2}}\left[\frac{-p F-(\tau F)_{1}}{\rho F+(\tau F)_{1}}\right]_{\alpha_{1}: \alpha_{2}}=-\frac{\alpha_{1}}{\alpha_{2}}=\alpha
$$

d. h. man hat, wenn man der Kürze wegen

setzt

$$
-f(x y)=\Theta
$$

$$
\Theta^{2}(x y)=\alpha .
$$

Da man durch Adjungierung der Wurzeln einer Resolvente dritten Grades, deren Form ich später ableiten werde, die Covariante $\tau$ in drei quadratische Formen $\varphi_{1}, \varphi_{2}, \varphi_{3}$ zerlegen kann, so kann man $F$ als Differenz zweier dieser Formen darstellen und somit die Gleichung $F=0$ lösen. Nun habe ich (l. c.) bewiesen, dass immer

$$
\frac{\frac{\partial F}{\partial y}(-f)}{\frac{\partial F}{\partial x}(-f)}=\frac{\frac{\partial P}{\partial y} F+Q x}{\frac{\partial P}{\partial x} F-Q y}
$$

ist, wo $P$ and $Q$ Covarianten von $F$ sind. Für $n>4$ kann nicht $P$ die Covariante $\tau$ sein, denn die Gleichung

$$
(n-1)^{2}=3 n-7+n
$$

hat nur die Lösungen 2 und 4. Wir haben also den Satz:

Es gibt keine algebraische Form von höherer als der vierten Ordnung, deren Covariante $\tau$ gleich Null gesetzt eine Abel'sche Gleichung von der Beschaffenheit ist, dass die rationale Function, mittelst deren sich alle Wurzeln durch eine derselben ausdrücken, gleich dem Quotienten der ersten Differentialquotienten der Grundform ist, oder, was dasselbe ist, deren Covariante $\tau$ der Gleichung 
genügt.

$$
\tau(-f)=\tau . \Phi
$$

Nun wäre es möglich, dass die associierte Form von $P$ den Factor $P$ enthält, dass also die Identität besteht

$$
P(-f)=P . \Psi,
$$

es wäre also $P=0$ eine Abel'sche Gleichung. Aus der Formel (40) folgt aber, dass die Periode derselben gleich 2 sein müsste und dass daher

$$
P=p_{1} \cdot \rho_{2} \ldots \frac{p_{\nu}}{2}
$$

wäre, wenn $v$ die Ordnung von $P$ ist. Würde sich $F$ durch die Differenz zweier der $\varphi$ darstellen lassen, so würde nach den obigen Auseinandersetzungen folgen, dass die conjugierte Formen sind und dass infolge dessen $P$ eine specielle Form von der vierten, sechsten oder zwölften Ordnung ist. Für $v=6$ ist $n=4$ und für $\nu=12$ ist $n=5$, im ersten Falle ist $P$ die Covariante $\tau$ und im zweiten kann unmöglich $F$ als Differenz zweier o dar. gestellt werden.

\section{\$. $\%$.}

Um einige Grenzfälle behandeln zu können, die, so viel mir bekannt ist, noch nicht erledigt sind, wird es nöthig sein, neue Beziehungen zwischen den Wurzeln der Lagrange'schen Resolvente und denjenigen von $\tau=0$ herzustellen. Zu diesem Zwecke bilden wir die Resolvente, welche die Summe je zweier zu einander gehörender Wurzeln von $\tau=0$ zu Wurzeln hat. Man sieht leicht ein, dass der erste und zweite Coefficient dieser Resolvente einen gemeinschaftlichen Factor besitzen müssen, so dass der Quotient derselben $\tau_{1}: \tau_{0}$ ist, wenn man die Coefficienten durch denselben Buchstaben mit angehängten Indices bezeichnet. Zu dieser Resolvente gelangt man offenbar durch die Substitution:

in die Resolvente

$$
\frac{H_{1}+2 \lambda F_{1}}{2\left(H_{0}+\lambda F_{0}\right)}=-z
$$

$$
\lambda^{3}-\frac{i}{2} \lambda--\frac{j}{3}=0 \text {. }
$$

Die Wurzeln dieser Gleichung sind demnach, da

$$
\lambda=-\frac{2 H_{0} z+H_{1}}{2\left(F_{0} z+F_{1}\right)}
$$

ist, rationale Functionen der Summen je eines zusammengehören den Paares der Wurzeln von $\tau=0$. Die Ausführungen der Sub stitution ergibt die Resolvente in folgender merkwürdigen Gestal 


$$
\text { Resolv. }=\tau_{0}\left\{\tau_{0} z^{3}+\tau_{1} z^{2}+\frac{2}{5} \tau_{2} z+\frac{1}{10} \tau_{3}\right\}=0 .
$$

Wir sind nun in der Lage, die Grenzfälle, in welchen einige der Coefficienten von $\tau$ verschwinden zu discutieren. Rückt eine Wurzel von $\tau=0$ ins Unendliche, so thut dies auch eine Wurzel der Resolvente (43) und eine Wurzel von (24) wird nach (42)

$$
\lambda=-\frac{H_{0}}{F_{0}^{\prime}} \text {. }
$$

Es handelt sich jetzt darum, das Verhalten der übrigen Wurzeln beider Resolventen zu ermitteln. Dazu dienen folgende Sätze:

\section{Satz I.}

Wenn $\tau_{0}=0$ ist, so ist die Resolvente (43) ein vollständiges Quadrat eines linearen Ausdruckes und dieser Ausdruck ist ein Factor von $\tau$.

$$
\text { Beweis. }
$$

Für $\lambda=-H_{0}: F_{0}$ muss

$$
H F_{0}-H_{0} F
$$

ein Quadrat sein. Nun ist, wie die Rechnung zeigt,

$$
H F_{0}-H_{0} F=\tau_{0} x^{3}+\tau_{1} x^{2} y+\frac{2}{5} \tau_{2} x y^{2}+\frac{1}{10} \tau_{3} y^{3}
$$

folglich ist im Falle $\tau_{0}=0$

$$
\begin{aligned}
H F_{0}-H_{0} F & =\left(\tau_{1} x^{2}+\frac{2}{5} \tau_{2} x y+\frac{1}{10} \tau_{3} y^{2}\right) y \\
& =(A x+B y)^{2} .
\end{aligned}
$$

Setzen wir

$$
\frac{H_{1}+2 \lambda_{1} F_{1}}{2\left(H_{0}+\lambda_{1} F_{0}\right)}=\frac{H_{1}+2 \lambda_{2} F_{1}}{2\left(H_{0}+\lambda_{2} F_{0}^{\prime}\right)}
$$

so gibt dies entwickelt die Gleichung

$$
\left(\lambda_{1}-\lambda_{2}\right)\left\{2 H_{0} F_{1}-H_{1} F_{0}\right\}=0,
$$

welche Identität in Wirklichkeit besteht, wenn $\tau_{0}=0$ ist. Wir erhalten daher in Bezug auf die ïbrigen Wurzeln von $\tau=0$ den

Satz II.

Die übrigen vier Wurzeln von $\tau=0$ theilen sich derart in zwei Paare, dass die Summe des einen gleich ist der Summe des anderen Paares. 


\section{Satz III.}

Im Falle $\tau_{0}=0$ sind entweder die übrigen vier Wurzeln alle oder es ist ein Paar derselben imaginär.

Die Formel

$$
\text { Beweis. }
$$

$$
\tau(-f)=\tau . \Phi
$$

besteht offenbar auch in diesem Falle und infolge dessen auch die Gleichungen (6) im §. 2. Die harmonische Lage der Wurzeln von $\tau=0$ ist also in nichts Anderem alteriert, als dass ein Element ins Unendliche rïckt. Das diesem zugeordnete Element muss nun in der Mitte zwischen zwei anderen Elementen liegen, wenn die vier Elemente eine harmonische Lage haben sollen. Es kann aber nicht ein Element zwei Strecken zweier zugeordneter Paare gleichzeitig halbieren, es folgt daher der Satz.

Es gibt noch eine Resolvente, welche zur Resolvente (24) in Beziehung steht and über den Fall Aufschluss gibt, wenn eine der Wurzeln von $\tau=0$ Null ist. Bilden wir nämlich die Resolvente, welche die Summe der reciproken je eines zusammengehörigen Paares von Wurzeln der Gleichung $\tau=0$, oder, was dasselbe ist, bilden wir die Resolvente von $x^{4} y^{4} \tau\left(\frac{1}{x}, \frac{1}{y}\right)=0$, so ist aus leicht fasslichen Gründen einzusehen, dass dieselbe die Gestalt hat:

$$
\tau_{6} z^{3}+\tau_{5} z^{2}+\frac{2}{5} \tau_{4} z+\frac{1}{10} \tau_{3}=0 \text {. }
$$

In dem Falle, wo eine Wurzel von $\tau=0$ Null wird rückt eine der Wurzeln der Resolvente (46) ins Unendliche. Es wird also in diesem Falle, da

$$
\left(\frac{1}{\alpha}+\frac{1}{\beta}\right)^{2}=\frac{\left(H_{1}+2 \lambda F_{1}\right)^{2}}{4\left(H_{0}+\lambda F_{0}\right)\left(\bar{H}_{4}+\lambda F_{4}\right)}
$$

ist, wenn $\alpha, \beta$ ein Paar zusammengehörender Wurzeln von $\tau=0$ sind,

$$
\lambda=-\frac{H_{4}}{T_{4}}
$$

Von dieser Resolvente gilt der

$$
\text { Satz IV. }
$$

Wenn $\tau_{6}=0$ ist, so ist

$$
\tau_{5} z^{2}+\frac{2}{5} \tau_{4} z+\frac{1}{10} \tau_{3}
$$

ein vollständiges Quadrat eines linearen Ausdruckes und die ser ist ein Factor von $\left(x^{4} y^{4}\right) \tau\left(\frac{1}{x}, \frac{1}{y}\right)$. 
Der Beweis wird ganz wie oben geführt. Es ist nämlich in diesem Falle

$$
H F_{4}-H_{4} F
$$

ein Factor von $\tau^{2}$ und muss deshalb ein vollständiges Quadrat sein. Nun ist aber, wie die Rechnung zeigt

$$
H F_{4}-H_{4} F=\tau_{6} y^{3}+\tau_{5} y^{2}+\frac{2}{5} \tau_{4} y x^{2}+\frac{1}{10} \tau_{3} x^{2}
$$

es ergibt sich daher der Satz.

Ebenso, wie in den Fällen $\tau_{0}=0, \tau_{6}=0$ die Bedeutung der Resolventen (43) und (46) verloren geht, so verlieren dieselben ihre Bedeutung im Falle $\tau_{3}=0$. Gegentheiligenfalls nämlich würde folgen, dass in diesem Falle die Gleichung $\tau=0$ zwei gleiche und entgegengesetzte Wurzeln hat. Dass dies nicht der Fall sein kann, zejgt man auf zweierlei Art.

Aus der Gleichung

$$
\alpha+\beta=-\frac{H_{1}+2 \lambda F_{1}}{2\left(H_{0}+\lambda F_{0}\right)}=0
$$

würde dann folgen, dass die Resolvente (24) die rationale Wurzel

$$
\lambda=-\frac{H_{1}}{2 F_{1}}
$$

hat, was, wie man sich leicht überzeugt, nicht der Fall ist.

Es wäre ferner

$$
2 H F_{1}-H_{1} F
$$

ein Factor von $\tau^{2}$ und daher ein vollständiges Quadrat. Es ist aber, wie die Rechnung zeigt,

$$
2 H F_{1}-H_{1} F=-\tau_{0} \dot{x}^{4}+\frac{2}{5} \tau_{2} x^{2} y^{2}+\frac{2}{5} \tau_{3} x y^{3}+\frac{1}{5} \tau_{4} y^{3}
$$

and es müsste daher auch

$$
-\tau_{0} x^{4}+\frac{2}{5} \tau_{2} x^{2} y^{2}+\frac{1}{5} \tau_{4} y^{4}
$$

ein vollständiges Quadrat eines quadratisehen Ausdruckes von der Form

$$
(x-A y)(y+A y)
$$

der ein Factor von $\tau$ ist, sein, was, wie man sich leicht überzeugt, nicht der Fall ist. Wir schließen daher, dass auch in diesem Falle die Gleichungen (43) und (46) keine Resolventen vorstellen.

Aus den Identitäten

$$
\text { ร. } 8 .
$$

$$
\begin{aligned}
& \tau_{1} x^{2}+\frac{2}{5} \tau_{2} x y+\frac{1}{10} \tau_{3} y^{2}=(A x+B y)^{2} \\
& \tau_{5} x^{2}+\frac{2}{5} \tau_{4} x y+\frac{1}{10} \tau_{3} y^{2}=\left(A^{\prime} x+B^{\prime} y\right)^{2}
\end{aligned}
$$


schließt man, dass die Discriminanten der linken Seiten $\tau_{0}$ resp. $\tau_{6}$ als Factoren enthalten muissen. In der That findet man leicht die Identität

$$
\left\{\begin{array}{l}
\frac{1}{10} \tau_{1} \tau_{3}-\left(\frac{1}{5} \tau_{2}\right)^{2}=\frac{1}{5} \tau_{0} \tau_{4} \\
\frac{1}{10} \tau_{3} \tau_{5}-\left(\frac{1}{5} \tau_{4}\right)^{2}=\frac{1}{5} \tau_{2} \tau_{6} .
\end{array}\right.
$$

Im Falle $\tau_{0}=0$ besteht, wie man sich leicht durch Rechnung überzengt, die Identität

$$
\tau_{2} \tau_{4}=\frac{1}{50} \tau_{3}^{2} \text {. }
$$

leiten :

Mit Hilfe dieser Formel lässt sich leicht folgender Satz abIm Falle $\tau_{0}=0$ hat die biquadratische Gleichung zwei reelle und zwei imaginäre Wurzeln, wenn $\tau_{1}$ und $\tau_{5}$ dasselbe Vorzeichen haben.

Es ist nämlich

$$
\text { Discrim. }(F)=\left(\tau \tau^{\prime}\right)^{6} \text {. }
$$

Für $\tau_{0}=0$ geht diese Formel über in

$$
\text { Discrim. }(F)=8 \tau_{2} \tau_{4}-20 \tau_{1} \tau_{5}-3 \tau_{3}^{2} .
$$

Infolge der Formel (ら1) hat man also

$$
\text { Discrim. }(F)=-\alpha \tau_{1} \tau_{5}-\beta \tau_{3}^{2} \text {, }
$$

d. $h$. die Discriminante ist im Falle $\tau_{0}=0$ negativ, wenn $\tau_{1}$ und $\tau_{\tilde{b}}$ dasselbe Vorzeichen haben. Wenn dies aber der Fall ist, so hat bekanntlich die Gleichung die oben ausgesprochene Eigenschaft.

\section{s. 9.}

Die Zerlegung von $\tau$ in drei quadratische Factoren stellt sich in drei formell von einander verschiedenen Arten dar.

Es ist

$$
\begin{aligned}
& \tau=\sqrt{H+e_{1} F} \sqrt{H+e_{2} F} \sqrt{H+e_{3} F} \\
& \tau=\sqrt{\mu U-\nu V} \sqrt{\mu \varepsilon^{2} \bar{U}-\nu \varepsilon^{2} V} \sqrt{\mu \varepsilon^{2} U-\nu \varepsilon \nu} \\
& \tau=\Phi_{1} . \Phi_{2} . \Phi_{3} .
\end{aligned}
$$

Der ersten entspricht die Resolvente

$$
k^{3}-\frac{i}{2} k l-\frac{j}{3} l^{3}=0
$$

1) Siehe moine Arbeit „Über die associierten Formen ete." 1889. Verlag von Gèrold. 
der zweiten, in der $U$ und $V$ die bekannten Tetraederformen

bedeuten, die Resolvente

$$
\begin{aligned}
& U=H+\lambda_{1} F \\
& V=H+\lambda_{2} F
\end{aligned}
$$

$$
i k^{2}+2 j k l+\frac{i^{2}}{6} l^{2}=0
$$

and der dritten, in der $\Phi_{1} \Phi_{2} \Phi_{3}$ die folgende Form haben

$$
\begin{aligned}
& \Phi_{1}=\left(x-\alpha_{1}\right)\left(x-\theta\left(\alpha_{1}\right)\right)=x^{2}+\psi_{1}\left(y_{1}\right)+\psi_{2}\left(y_{1}\right) \\
& \Phi_{2}=\left(x-\alpha_{2}\right)\left(x-\Theta\left(\alpha_{2}\right)\right)=x^{2}+\psi_{1}\left(y_{2}\right)+\psi_{2}\left(y_{2}\right) \\
& \Phi_{3}=\left(x-\alpha_{3}\right)\left(x-\Theta\left(\alpha_{3}\right)\right)=x^{2}+\psi_{1}\left(y_{3}\right)+\psi_{2}\left(y_{3}\right)
\end{aligned}
$$

die Resolvente (43)

$$
\tau_{0} y^{3}+\tau_{1} y^{2}+\frac{2}{5} \tau_{2} y+\frac{1}{10} \tau_{3}=0 .
$$

Die Beziehungen zwischen den Wurzeln von (24) und (43) haben wir im $\$ .7$ kennen gelernt. Was die Beziehungen zwischen den Wurzeln von (54) und (24) anlangt, so können wir, da die Factoren in (I) und (II) einzeln übereinstimmen mixssen, schlielien, dass die Wurzeln von (24) sich durch algebraische Summen der Wurzeln von (54) ausdricken lassen. Wir haben daher den

Satz.

Die Gleichung (24) ist die Resolvente der Gleichung (54). 